International Mathematical Forum, 1, 2006, no. 35, 1729-1731

\title{
ON A PROBLEM OF EL-Metwally et al.
}

\author{
Jean M. Tchuenche ${ }^{1}$ and Nyimvua S. Mbare \\ Mathematics Department, University of Dar es Salaam \\ P.O.Box 35062, Dar es Salaam, Tanzania \\ shabanmbare@yahoo.com \\ Peter O. K. Aiyelo \\ Department of Mathematics and Computer Science \\ Igbinedion University, Okada, P.M.B. 0006, Benin City, Nigeria \\ aiyelo2000@yahoo.com
}

\begin{abstract}
This paper is an attempt to provide a solution to the open problem raised by EL-Metwally et al., (2001): that is; to determine the boundedness character of the positive solution of equation (1) when the population growth rate is an exponential function of the immigration rate. We make use of a dimensionless quantity, $\epsilon$, say, which has the characteristics of a threshold parameter.
\end{abstract}

Keywords: Boundedness, difference equation, population dynamics

Mathematics Subject Classification: 39A10, 39A11, 39A13

\section{Introduction:}

El-Metwally et al., in [1] studied the global stability, the boundedness nature, and the periodic character of the positive solutions of the difference equation

$$
x_{n+1}=\alpha+\beta x_{n-1} e^{-x_{n}} .
$$

This equation may be viewed as describing the dynamic of a population, in which case $\alpha$ represents the immigration rate, while $\beta$ is the population growth rate. This paper is an attempt to provide a solution to the open problem raised

\footnotetext{
${ }^{1}$ Correspondence: jmt_biomaths@yahoo.co.uk.
} 
therein, that is; to determine the boundedness character of the positive solution of equation (1) when $\beta=e^{\alpha}$.

Equation (1) is interesting in its own right, and thus worthy of study for the purpose of mathematical elucidation. Our analysis follows basically the approach adopted [1], with appropriate modifications.

\section{The Model Equation}

As pointed out by Metwally et al., in [1], if $\beta=e^{\alpha}$, this implies that the population growth rate is an exponential function of the immigration rate. Hence, an equation of the form

$$
x_{n+1}=\alpha+x_{n-1} e^{\alpha-x_{n}}, \quad n=0,1,2, \ldots
$$

may model the dynamics of insects population such as locusts invading a lowly population area.

Difference equations arise in modelling various life situation because animals and many plants are counted in discrete units. For instance, the wellknown logistic map arises in a variety of systems such as chemical reactions electrical circuits, hydrodynamical flows, and population dynamics. Another example is the modified Henon map

$$
\begin{aligned}
H_{n+1} & =a+y_{n}-b H_{n}^{2} \\
y_{n+1} & =c H_{n}
\end{aligned}
$$

For the classical Henon map, $a=1, b=1$ and $c=1.3$.

In general, stability is the necessary and sufficient condition for convergence of a properly posed initial value problem (and a finite difference approximation that satisfies the consistency condition).

Let $I$ be an interval of real numbers, and $f: I \times I \rightarrow I$ be a continuously differentiable function. Consider the difference equation

$$
x_{n+1}=f\left(x_{n}, x_{n-1}\right), \quad n=1,2, \ldots
$$

where the initial condition $x_{0}, x_{1} \in I$.

Since we are dealing with population dynamics, we do not consider $x_{-1}$, as in [1]. Also, $x_{n} \geq 0$, negative values of $x_{n}$ are biologically meaningless [3] and no one cares what these values do when $n$ is negative [2]. Also, negative time steps are also irrelevant.

The following comparison Theorem (which we state without proof) will be useful in the sequel.

Theorem 1: Assume that $\alpha \in \mathbb{R}^{+}$. Let $\left\{x_{n}\right\}_{n=1}^{\infty}$ and $\left\{z_{n}\right\}_{n=1}^{\infty}$ be two sequences of real numbers, such that:

$$
x_{0} \leq z_{0} \text { and } x_{1} \leq z_{1}
$$


and

$$
\left\{\begin{array}{l}
x_{n+1} \leq \alpha x_{n-1}+e^{\alpha} \\
z_{n+1}=\alpha z_{n-1}+e^{\alpha}
\end{array}\right.
$$

Then $x_{n} \leq z_{n}$ for $n \geq 0$.

Now the main result.

Theorem 2: Suppose $e^{\alpha}<\infty, 0<\alpha<1$, and let $\left\{x_{n}\right\}_{n=0}^{\infty}$ be a positive solution of equation (2) for $n \geq 1$, then $x_{n+1} \leq \alpha+x_{n-1}$

Proof: Consider $z_{n+1}=\alpha+\epsilon z_{n-1}, \epsilon>0$ is a dimensionless scaling parameter, $n=1,2, \ldots$ (5) with $z_{0}=x_{0}$ and $z_{1}=x_{1}$. Then $x_{n} \leq z_{n}$ for all $n \geq 0$.

Now,

$$
\lim _{n \rightarrow 0} z_{n}=\lim _{\epsilon \rightarrow 1} \frac{\alpha}{1-\epsilon}
$$

$\epsilon$ here is a threshold parameter. If $\epsilon=1$, there is a blow-up solution. Thus, if $\epsilon<1$, then, every positive solution of equation (2) is bounded. For $\epsilon>1$, positive solution of equation (2) will be negative since $\lim _{n \rightarrow \infty} z_{n}<0$, a contradiction. Thus, $\epsilon$ cannot exceed unity. By neglecting terms of order greater than or equal to 2 , we have that

$$
\lim _{\epsilon \rightarrow 1} \frac{\alpha}{1-\epsilon}=\lim _{\epsilon \rightarrow 1} \alpha\left(1+\epsilon-\frac{\epsilon^{2}}{2}-\cdots\right) \leq 2 \alpha
$$

This solution is locally stable but globally unstable as a small perturbation will disrupt the system. This crude approximation tells us that the sequence in (2) is bounded, if we can find a scaling parameter $\epsilon>0$, such that $z_{n+1}=\alpha+\epsilon z_{n-1}$. A possible guess for $\epsilon$ to be an optimum control is when $0<\epsilon<1$. Thus, every positive solution of (5) is bounded if $\epsilon<1$.

\section{References}

[1] El-Metwally, H., Grove, E.A., Ladas, G., Levins, R. and Radin, M. (2001). On the Difference Equation. $x_{n+1}=\alpha+\beta x_{n-1} e^{-x_{n}}$. Nonlinear Analysis 47, 4623, 4634 .

[2] Rosen, R. (1993) On Models and Modelling. Appl. Math. Comput. 56, 359-372.

[3] Watson, E.J. Laplace Transforms and Applications. New York: von Nostrand Reinhold, 1981.

\section{Received: June 5, 2006}

